Revue d'histoire de l'Amérique française

\#AS REVUE D.HISTOIRE DE L'AMÉRIQUE FRANÇAISE

\title{
Deux sermons de M. J.-J. Lartigue, p.s.s., lors de la guerre de 1812
}

\section{François Beaudin}

Volume 22, numéro 2, septembre 1968

URI : https://id.erudit.org/iderudit/302783ar

DOI : https://doi.org/10.7202/302783ar

Aller au sommaire du numéro

Éditeur(s)

Institut d'histoire de l'Amérique française

ISSN

0035-2357 (imprimé)

1492-1383 (numérique)

Découvrir la revue

Citer ce document

Beaudin, F. (1968). Deux sermons de M. J.-J. Lartigue, p.s.s., lors de la guerre de 1812. Revue d'histoire de l'Amérique française, 22(2), 301-308.

https://doi.org/10.7202/302783ar d'utilisation que vous pouvez consulter en ligne.

https://apropos.erudit.org/fr/usagers/politique-dutilisation/ 


\section{DOCUMENTS INÉDITS}

\section{DEUX SERMONS DE M. J.-J. LARTIGUE, p.s.s., LORS DE LA GUERRE DE 1812 \\ INTRODUCTION}

Monseigneur Pierre Denaut, évêque de Québec, ne voulut jamais consentir à se priver des services de l'abbé Jean-Jacques Lartigue, son secrétaire, le futur évêque de Montréal, quoique ce dernier lui eût souvent demandé la permission d'entrer à St-Sulpice. Ce n'est qu'après la mort de Mgr Denaut, le 17 janvier 1806, que l'abbé Lartigue put réaliser son rêve. Le ler février 1807, il était agrégé comme directeur du Séminaire de Montréal.

L'auteur de la biographie de Mgr Lartigue que nous trouvons au début du volume I des Mandements des Evêques de Montréal, nous parle de "son rare talent pour la prédication". Un événement survenu au moment de la guerre de 1812 lui permit de mettre ce talent à profit: "Le gouvernement n'eut qu'à se féliciter de son habileté à manier les esprits de ses concitoyens, pendant la dernière guerre américaine. Car, un certain Légiste, d'origine britannique, ayant essayé de persuader aux milices canadiennes, alors sur pied, qu'on ne pouvait pas légalement les retenir au-delà d'une certaine époque, elles menaçaient de se débander. Sur l'invitation du gouverneur général, Sir Georges Prévost, M. Roux se décida à envoyer un des Messieurs de sa maison pour retenir ces braves miliciens dans la ligne de leur devoir. Le choix du Supérieur tomba sur M. Lartigue qui n'eût pas plutôt paru au milieu de ses chers concitoyens que l'ordre fut à l'instant rétabli."' 1

Monsieur Jean-Pierre Wallot nous a présenté, dans son travail intitulé "Une émeute à Lachine contre la conscription 1812" " tout le contexte de cette question. Il sera utile, pensonsnous, d'ajouter au dossier relatif à cette émeute deux pièces dignes d'intérêt.

Le travail entrepris, en septembre 1967, par le P. Gilles Chaussé, s.j., aux Archives de la Chancellerie de l'Archevêché de Montréal, en vue de la thèse de doctorat qu'il rédige sur Mgr Lartigue, lui a permis de découvrir le texte de deux sermons

${ }^{1}$ Mandements des Evêques de Montréal, I: VIII.

2 Voir RHAF, XVIII, no 1 (juin 1964) : 112-137 et no 2 (septembre 1964) : 202-232. 
prononcés par M. Lartigue, l'un, à Pointe-Claire, le 5 juillet 1812 , et l'autre, à Lachine, le 12 juillet 1812 .

Ajoutés à la lettre circulaire de Descheneaux, v.g. de Québec, à tous les curés ${ }^{3}$ et au Mandement de M. J.-H.-A. Roux, p.s.s., v.g., pour la paroisse de Montréal ${ }^{4}$, ces textes donnent un bon aperçu de la mentalité du clergé, en 1812, face à l'autorité établie.

D'autre part, en ce qui concerne Mgr Lartigue, ils nous permettent un retour de 25 ans en arrière: la doctrine exprimée par Mgr Lartigue dans son Mandement sur les troubles de 1837 ne sera pas une nouveauté, une réaction de peur face à l'événement; en 1812, il avait exprimé la même pensée, en chaire, alors qu'il n'était âgé que de 35 ans.

On remarquera la construction et l'élan de ces textes. Lartigue parle d'abord du moment agité qu'il vit et de l'autorité qu'il possède pour s'adresser à ses paroissiens. Ensuite il développe son argumentation à partir de trois points: l'honneur dû au nom canadien, la reconnaissance pour le Gouvernement paternel qui règne sur le pays, enfin le respect dû à la Religion qui ordonne, en tout temps mais surtout en temps de guerre, une obéissance parfaite au Roi et à tous ceux qui commandent de sa part. Voilà la structure fondamentale de ces deux textes.

Un aspect intéressant nous est révélé par le deuxième de ces sermons. Il semblerait qu'un des motifs du refus des conscrits d'aller se battre ait été le fait que des Français faisaient partie de l'armée américaine. Lartigue ne dit-il pas, en effet: "Et quand même il se trouverait contre vous quelques-uns de ceux qui sont descendus du même sang que vous, de ces François modernes, si prodigieusement dégénérés de leurs Pères, et qui n'ont plus avec vous d'autre lien que celui de l'humanité, vous ne laisserez pas de les combattre et de les vaincre, parce qu'ils seront les ennemis de votre Roi et les vôtres !"

Quoi qu'il en soit, l'Eglise canadienne a dû se sentir dans un bien grand état de faiblesse, de précarité et d'insécurité, en ces débuts du $19 \mathrm{e}$ siècle, pour mettre une telle insistance à appuyer l'ordre établi et utiliser autant de persuasion pour amener ses fidèles à prendre les armes, comme en font foi la lettre circulaire de Descheneaux, le Mandement de M. Roux, et les deux sermons qu'on lira maintenant.

21 août 1968

FrançoIs BEAUdIN, ptre Archiviste

Archevêché de Montréal

3 Mandements des Evêques de Québec, 3: 86-88.

4 Mandements des Evếques de Québec, 3: 88-91. 


\section{SERMON DE M. LARTIGUE, p.s.s. ${ }^{5}$}

5 juillet, 1812 A la Pte Claire.

En quelle Paroisse, M.F. \& dans quelles circonstances me vois-je aujourd'hui obligé d'ouvrir la bouche pour vous tracer fidèlement la ligne de vos devoirs? Dans une Paroisse qui vient d'être agitée des plus violentes convulsions, \& qui a été témoin de l'égarement de plusieurs de ses habitants: dans des circonstances lamentables, où plusieurs d'entre vous ont à gémir sur l'erreur coupable de leurs amis ou de leurs proches. Personne en ce moment ne prend plus de part que moi à votre juste affliction \& plût à Dieu que ma faible voix pût parvenir aux pieds de l'homme généreux et noble qui nous gouverne! Je tâcherois de l'attendrir sur le sort de ces hommes, criminels, il est vrai, \& grandement criminels, mais encore plutôt égarés que rebelles, \& moins coupables que les conseillers pervers qui les ont poussés dans l'abyme. Mais un devoir plus pressant m'occupe tout entier, un de mes devoirs les plus sacrés, celui de vous prêcher la fidélité, l'amour, l'obéissance que vous devez à votre légitime souverain \& au Gouvernement bienfaisant qui protège cette Province. M.F. Rien dans moi ne peut vous être suspect: je suis, quoique indigne, Ministre de J.C., \& sans doute qu'en vous parlant en son nom je ne voudrois pas vous tromper: je n'ai jamais rien reçu du Gouvernement, \& tous ceux qui me connaissent savent bien que je n'en attends rien, sinon sa protection qui est commune à tous les sujets de Sa Majesté: vous êtes Canadiens; je le suis aussi, et nos véritables intérêts ne peuvent être séparés. Eh bien! mes chers compatriotes, pour l'honneur de cette brave nation Canadienne à laquelle nous nous glorifions d'appartenir, par reconnaissance pour le Gouvernement paternel qui nous protège depuis tant d'années, \& à qui seul, après Dieu, nous devons le bonheur constant dont nous avons joŭis jusqu'à ce jour, par respect surtout pour votre auguste Religion qui vous ordonne en tout temps, mais principalement dans la crise actuelle de la guerre, une obéissance parfaite au Roi \& à tous ceux qui vous commandent de sa part; par tant de motifs réunis, montrez-vous ce que vous devez être, c.-a.-d. des sujets fidèles et loyaux, prêts à verser votre sang pour l'honneur de votre Roi, comme pour la gloire de votre Reli-[2]gion. Je dis que vous devez vous montrer loyaux pour l'honneur de votre Pays. Et quelle nation fut jamais plus vaillante, plus généreuse, plus fidèle à son Prince que la Nation Canadienne? Auriez-vous donc oublié comme vos ancêtres et plusieurs mêmes d'entre vous se distinguerent en 75 contre ces mêmes ennemis que vous aurez encore à combattre? Auriez-vous perdu la mémoire des généreux efforts que fit alors la partie saine des Canadiens pour repousser ces voisins rebelles à leur Souverain légitime, \& qui les obligerent à retourner honteusement dans leur pays ? Auriez-vous dégénéré du courage de vos ayeux jusqu'au point de craindre des ennemis que vous avez déjà vaincus, tandis que depuis cette époque Le Canada a au moins redoublé en nombre, en force $\&$ en moyens de défense et d'attaque ? Non, je m'en repose sur mes compatriotes, il se sera pas dit qu'ils auront aimé leur bon Roi, moins que nos pères, moins que ces braves Canadiens qui ont versé leur sang pour sa gloire: non, il ne sera pas dit qu'ils auront perdu tout le courage qui les distinguoit autrefois, \& qu'ils n'auront eu d'énergie

5 Original conservé aux Archives de la Chancellerie de l'Archevêché de Montréal, Dossier 901.039. Les chiffres entre crochets indiquent la pagination de l'original. 
que pour la révolte. La reconnaissance seule envers la Mère-patrie devroit suffire pour vous instruire de votre devoir là-dessus. Quelle Nation eut jamais autant de droit à la gratitude d'un autre peuple que la Nation Angloise en a par rapport à vous ? Depuis plus de 50 ans que vous êtes soumis à son empire, quels bienfaits n'en avez-vous pas reçus ? Votre Sainte Religion autorisée, vos Pasteurs respectés, vos anciennes loix conservées, vos propriétés, vos biens, vos personnes garanties et protégées, votre commerce, votre industrie encouragés, voilà une petite partie des biens que vous devez au Gouvernement protecteur qui vous a conquis; \& tous ces biens, vous en avez joŭi tandis que le reste du monde étoit depuis plus de 20 ans accablé de tous les maux qu'une guerre longue $\&$ meurtrière peut produire: vous viviez dans la paix \& la tranquilité, sans impôts, sans inquiétude, \& sans allarmes, tandis que l'Angleterre épuisoit son sang \& ses trésors pour vous protéger; \& lorsqu'elle supportoit elle-seule toutes les charges d'une guerre rŭineuse \& cruelle, elle ne vous demandoit pour récompense qu'une fidélité à toute épreuve. Après cela, si jamais vous étiez infidèles à cette Nation généreuse n'auroit-elle pas le droit de vous adresser les mêmes reproches que Dieu fesoit à son peuple: qu'ai-je pû faire pour vous que je n'aye pas fait, quid potui facere \& non feci ? Mais ici la voix de la Religion s'éleveroit encore bien plus fort pour vous prêcher votre devoir. Car il n'est plus temps de dire que vos Pasteurs doivent vous annoncer l'Evangile sans se mêler d'affaires politiques. Non, M.F., quand nous vous parlons de vos obligations comme sujets \& comme citoyens, ce n'est pas autre [3] chose que l'Evangile, \& le pur Evangile que nous vous prêchons. Depuis quand les devoirs des sujets envers leurs souverains ne sont-ils donc plus un dogme de la Religion. Trouve-t-on à redire que nous nous mêlions des affaires de famille, quand nous prescrivons aux époux leur devoirs envêrs leurs époux, ceux des enfans envêrs leurs parents, ceux des parents envêrs leurs enfans ? Est-ce que la Loi de J.C. n'a pas réglé toutes les obligations de la grande famille qui est l'Etat, comme celle des familles particulières qui le composent? N'avez-vous donc jamais entendu ces oracles sacrés de la Loi de Dieu: Rendez à César ce qui est à César: que toute ame soit soumise aux puissances établies de Dieu; car celui qui résiste à la puissance, résiste à Dieu lui-même? Avez-vous oublié ces paroles du Prince des Apôtres: soyez soumis à tous ceux qui sont au dessus de vous, soit au Roi comme au Chef de l'Etat, soit aux Gouverneurs comme à ceux qui vous commandent de sa part: \& encore soyez soumis à tous ceux qui vous gouvernent, quand même ils seroient injustes à votre égard, car c'est là la volonté de Dieu ? Voilà, M.F. l'Evangile dans toute sa pureté: ce n'est pas ici la politique qui nous inspire ces maximes; c'est la parole même de votre Dieu que nous annonçons de sa part. Paroles si biencomprises par les vrais Fidèles, qu'il était inoŭi qu'il y eût jamais, je ne dis pas aucune révolte, mais la plus légère désobéissance aux Empereurs parmi les premiers Chrétiens: \& cependant alors ces Empereurs étaient Payens; et ils persécutoient à outrance le Christianisme. Aussi Tertulien qui vivoit au $2 \mathrm{~d}$ siècle de l'Eglise, temps où la Religion s'étendoit déjà au delà des bornes de l'Empire Romain; Tertulien, dis-je, défioit les Payens de citer un seul Chrétien qui se fut rendu rebelle à son Prince. 0 ! beaux jours de la Religion, temps heureux ou un Chrétien auroit été censé avoir apostasié de sa Religion en manquant de fidélité à son Prince, jours de gloire pour les vrais fidèles, qu'êtes-vous donc devenus ? Nous les reverrons encore, M.F. ces beaux jours; du moins je l'espère de votre piété et de votre bravoure, si un ennemi étranger ose mettre le pied sur votre territoire. Oui, c'est alors que vous payerez de votre bras, de vos biens, de votre sang même, s'il le faut, pour la défense de votre patrie \& de votre Roi: alors, 
vous prouverez que vous êtes les dignes enfans de ces braves Canadiens qui courroient aux combats comme à un jour de fête. Oui, vous vous montrerez guerriers comme eux, comme eux attachés au Gouvernement sans lequel vous avez le bonheur de vivre; \& quand même il se trouverait parmi vos ennemis [4] quelques uns de ceux qui sont descendus du même sang que vous, vous ne laisserez pas de les combattre et de les vaincre, parcequ'ils seront les ennemis de votre Roi \& les vôtres. Ce Roi à qui seul vous devez l'obéissance, c'est George Trois, votre légitime souverain; \& il semble que l'Eglise veuille nous engager à lui renouveller l'assurance de votre dévoǔement en nous fesant lire dans l'Office de ce jour ces belles paroles, Vivat Rex in eternum. Que chacun de vous répète donc avec moi, autant de coeur que de bouche: Vive le Roi.

Repleti sunt omnes spiritu Sancto. Ils furent tous remplis du St-Esprit. 6 Ces paroles sont tirées de l'Epître de ce jour, aux Actes des Apôtres, ch.2d. Plût à Dieu, M.F. qu'on pût dire de chacun de vous dans cette grande solemnité, ce que l'Ecriture nous apprend de tous les Disciples renfermés dans le Cénacle: Ils ont tous été remplis du St-Esprit: Repleti sunt omnes Spiritu Sancto. L'Esprit Saint en descendant, à pareil jour, dans le coeur des Apôtres, les changea en des hommes tout nouveaux: mais quel changement a-t-il opéré jusqu'à ce moment dans vos coeurs ? Depuis la dernière fois que $j$ 'eus le plaisir de vous parler publiquement, beaucoup de changements ont eû lieu dans le monde: vous avez changé vous-même sous plusieurs rapports, ne fût-ce que par la rapidité du temps qui vous pousse de jour en jour vêrs le tombeau; mais ce changement si désirable, ce changement du coeur, cette conversion solide, en un mot, ces effets de l'Esprit-Saint qui doivent vous rendre de bons Chrétiens \& des guerriers intrépides, les avez-vous ressentis. Je m'arrête à cette dernière réflexion qui embrasse vos devoirs comme Miliciens; \& pour réveiller en vous les sentiments de cette ardeur militaire qui doit vous animer plus que jamais dans ces moments critiques, je vous en conjure, pour l'honneur de cette brave nation Canadienne, \&c. comme au commencement.

II

\section{SERMON DE M. LARTIGUE, p.s.s.7}

12 juillet, 1812. A la Chine.

Vidi sanctam Civitatem, Jerusalem novam, paratam sicut sponsam ornatam viro suo. Je vis la ville sainte, la nouvelle Jérusalem, ornée comme une épouse qui se pare pour son époux. Ces paroles sont tirées de l'Epitre de ce jour. Au Ch. 21e de l'Apocalypse, V.2d.

Ce n'est pas seulement de la Jérusalem céleste, de cette cité sainte dont les Anges sont les habitants \& dont le Seigneur est la lumière, que St.Jean a voulu nous peindre la magnificence dans ses Révélations; c'est à nos Temples, dont nous célébrons en ce jour la Dédicace, que l'Eglise applique aussi ces belles paroles: ce sont eux qui, consacrés au Très-haut avec l'appareil des plus augustes cérémonies, méritent nos plus profonds

${ }^{6}$ Ce paragraphe semble être une autre introduction, pour un autre dimanche, au même sermon.

7 Original conservé aux Archives de la Chancellerie de l'Archevêché de Montréal, Dossier 901.039. 
respects, à cause du Dieu qui y habite: ce sont eux où nous avons été régénérés dans les eaux sacrées du baptême, purifiés tant de fois dans le bain salutaire de la pénitence, nourris de la chair de l'agneau sans tache: ce sont eux enfin où Dieu nous a fait souvent entendre sa parole dans la chaire de vérité, où le Sang de J.C. à rougi nos autels dans le St. Sacrifice, où nos cendres inanimées seront encore portées après notre mort pour $y$ recevoir les honneurs de la Sépulture. Que de titres, M.F. nous attachent donc à nos Eglises, à ces aziles sacrés où le riche comme le pauvre, le Prince \& le sujet, les grands comme les petits, trouvent, auprès du Père commun qu'ils viennent $y$ adorer, un secours dans leurs besoins, de la consolation dans leurs peines, tous les biens, en un mot, pour le temps \& pour l'éternité ! Mais, ô mon Dieu ! quelle pensée déchirante vient ici troubler ma raison \& navrer mon coeur ? Ces temples dédiés à votre gloire $\&$ si chers à votre peuple, seroit-il donc possible que nous les verrions un jour, sous peu de temps peut-être, prophanés, pillés, incendiés, effacés de dessus la face de la terre? Seroit-il possible qu'un ennemi jaloux du bonheur que nous possédons voulût nous le ravir, \& qu'en nous enlevant nos biens, notre repos, notre liberté \& nos vies, il plaçât l'abomination de la désolation jusque dans votre sanctuaire ? O idée accablante! ô funeste pressentiment! Ah ! Seigneur, punissez-nous puisque nous le méritons, mais châtiez-nous dans votre miséricorde: Si le fléau terrible de la guerre n'est pas trop pour vous venger de nos crimes, nous adorerons en gémissant la main paternelle qui nous frappera; mais au moins laissez-nous nos temples, ces lieux sacrés où nous pourrons encore vous fléchir: [laisseznous nos autels, pour y immoler tous les jours la victime de propitiation: laissez-nous la Religion sainte qui seule peut nous consoler au milieu de nos malheurs.] 8 Il me semble, Xtiens, que mes voeux sont exaucés, quand je vois cette troupe de guerriers généreux, prêts à lever le bras pour défendre le sanctuaire: \& me confiant en celui qui donne quand il lui plaît la victoire au petit nombre sur des milliers d'ennemis, je me dis à moi-même: non, le Seigneur ne permettra pas qu'une nation si brave \& si catholique devienne la proye de ces infidèles orgueilleux: il ne souffrira pas que la gloire du nom Anglois qui nous protège soit ternie dans ce petit coin du monde, tandis qu'elle sert de boulevard à la [?] 9 de tant d'autres peuples: il ne laissera pas mes compatriotes, [2] ces braves Canadiens, se dégrader de leur caractère national, se rendre coupables d'ingratitude envêrs la Mere-Patrie, \& méconnoître les devoirs les plus sacres de la Religion. Oui, M.F. je le répète, manquer, en ce moment critique, de loyauté \& de courage, ce serait souiller d'une tache ineffaçable l'honneur national. Et quelle Nation fut jamais plus vaillante, plus propre aux dangers des combats, plus dévouée à son Prince, que la nation Canadienne? Auriez-vous donc oublié comme vos ancêtres, \& plusieurs même d'entre vous, se distinguèrent en 75 contre ces ennemis que vous aurez encore à combattre ? Auriez-vous perdu la mémoire des généreux efforts que fit alors la partie saine des Canadiens pour repousser ces voisins rebelles à leur Souverain légitime, \& les forcer de retourner honteusement dans leur pays ? Auriez-vous dégénéré du courage de vos ayeux, jusqu'au point de craindre des hommes que vous avez déjà vaincus, tandis que depuis cette époque le Canada a aumoins redoublé en nombre, en forces, $\&$ en moyens de défense ou d'attaque ? Quel deshonneur au nom Canadien l'original.

8 Tout ce qui est entre crochets, qui sont de nous, est en marge dans

9 Un mot illisible. 
[à ce nom dont nous sommes si glorieux] 10, si nous montrions moins d'empressement, moins de bonne volonté que nos Co-sujets Britanniques pour la défense de nos foyers, \& la gloire de notre Roi ! Quelle honte pour nous, Ministres de l'Evangile, si notre voix, que vous avez si bien entendue en d'autres occasions, si [sic] trouvoit impuissante quand il s'agit de vous persuader de vos obligations comme sujets \& comme citoyens ! Il ne nous resteroit plus alors qu'à embrasser nos autels en déplorant votre aveuglement, \& à nous renfermer dans nos temples pour être ensevelis sous leurs rŭines. Mais non, je m'en repose sur mes compatriotes, il ne sera pas dit qu'ils auront aimé leur bon Roi moins qu'aucun de ses autres sujets; il ne sera pas dit qu'ils auront rien perdu de ce beau feu militaire qui les distinguoit autrefois; il nessera pas dit qu'ils se seront couverts d'un opprobre éternel par leur ingratitude envêrs la Mere-patrie. Car enfin, quelle nation eut jamais autant de droit à la reconnaissance d'une autre peuple que la Nation Angloise en a par rapport à vous? Depuis plus de 50 ans que vous êtes soumis à son empire, quels bienfaits n'en avez-vous pas reçus? votre sainte Religion autorisée, vos Pasteurs respectés, vos anciennes loix conservées, vos propriétés, vos biens, vos personnes garantis et protégés, votre commerce, votre industrie encouragés, voilà une petite partie des faveurs dont vous êtes redevables à la nation généreuse qui vous a conquis; \& tous ces avantages, vous en avez joŭi tandis que le reste du monde était depuis plus de 20 ans accablé de tous les maux qu'une guerre longue \& meurtrière peut produire: vous viviez à l'ombre de la protection [de la Grande-Bretagne] 11, dans la paix \& la tranquillité, sans impôts, sans inquiétudes \& sans allarmes, tandis qu'elle épuisoit son sang $\&$ ses trésors pour détourner de ce pays les ravages du torrent révolutionnaire [3] qui engloutissoit tant d'autres peuples; \& lorsqu'elle-seule supportoit toutes les charges d'une guerre rŭineuse \& cruelle, elle ne vous demandoit pour récompense qu'une fidélité à toute épreuve. Tout bienfait impose à celui qui l'a reçu le tribut de la reconnaissance: que sera-ce donc quand il s'agit de graces aussi signalées, aussi multipliées ? Sans doute les Canadiens se piquent autant de générosité que de bravoure; \& vous rougiriez apparament de rester en arrière à l'égard d'une Nation aussi noble \& aussi magnanime. Eh bien ! M.F. le moment est arrivé de vous acquitter envêrs la Mere-patrie; voici l'occasion favorable, non pas de payer tout ce que vous lui devez (ce seroit exiger au delà de votre pouvoir) mais de témoigner au-moins le désir que vous avez de la satisfaire. Quelques efforts que vous fassiez, elle en fera encore beaucoup plus que vous: tandis que vous défendrez courageusement vos frontières, des flottes triomphantes parcoureront les mers pour assurer une correspondance nécessaire entre elle \& nous; ses régiments nombreux \& aguerris traverseront l'océan pour voler à notre secours, \& attaquer jusque dans ses ports \& ses forteresses notre ennemi commun; le sang Anglois coulera dans nos plaines: \& remarquez-le bien, mes chers compatriotes, ce ne sera pas pour protéger les Iles Britaniques, qui sont à l'abri de toute attaque; mais ce sera pour défendre vos femmes, vos enfans, vos parents, vos propriétés, votre Religion, vos temples, en un mot, tout ce qui vous est le plus cher: voilà comme l'Angleterre comble de nouveaux bienfaits un peuple qui sait reconnoître les anciens. Mais quand l'honneur, la gratitude, tous les motifs ne se réuniroient pas ici pour vous persuader, la voix seule de la Religion, cette voix si puissante sur de bons chrétiens \& de vrais Catholiques, ne vous prêcheroit-elle pas encore plus hautement votre devoir. Ici, Xtiens,

10 En marge, dans l'original.

11 En marge, dans l'original. 
ce n'est plus le langage des hommes que je dois vous tenir, mais c'est de la part du Dieu des armées, de ce Maître souverain dont je suis le Ministre, malgré mon indignité; c'est en son nom que je vous dis avec le Grand Apôtre: que toute ame soit soumise aux puissances établies de Dieu; car celui qui résiste à la puissance résiste à Dieu lui-même. C'est avec le Chef des Apôtres que je vous dis: Soyez soumis à tous ceux qui sont au dessus de vous, soit au Roi comme au chef de l'Etât, soit au Gouverneur comme à celui qui vous commande de sa part; $\&$ encore: soyez soumis à tous ceux qui vous gouvernent, quand même ils seraient injustes à votre égard, car c'est là la volonté de Dieu. Quelle foule de textes de l'Evangile n'aurois-je pas à vous produire, si j'entreprenois de détailler tous les devoirs des sujets envers leurs Souverains ? Mais qu'est-il besoin d'insister sur tant d'oracles des divines Ecritures, quand nous les voyons si bien-expliqués [4] par la conduite des premiers Fidèles ? Pendant 300 ans de persécution ouverte contre le Christianisme, vit-on jamais parmi les Chrétiens, je ne dis pas aucune révolte, mais la plus legere désobéissance aux Princes payens, auteurs de cette persécution? Ne vit-on pas des légions entières offrir de marcher contre des ennemis de l'Empire, lors-même qu'on employoit la force des tourments pour les faire renoncer à la Foi. Tertulien ne défioit-il pas tous les Payens de son temps de citer un seul Chrétien infidèle à son serment, \& qui ne fût prêt à verser tout sang [sic] pour la gloire des Empereurs qui les persécutoient? 0 ! beaux jours de la Religion, temps heureux où un chrétien auroit été censé avoir apostasié en manquant de fidélité à son Prince, jours de gloire pour les vrais Fidèles, qu'êtes-vous donc devenus? Nous les reverrons encore, M.F. ces beaux jours, (dumoins je l'attends de votre piété \& de votre valeur) si un ennemi étranger ose mettre le pied sur votre territoire. Oui, c'est alors que vous saurez payer de vos bras, de vos biens, de votre sang même, s'il le faut, pour le salut de votre patrie \& la gloire de votre Roi: alors, vous prouverez que vous êtes les dignes enfans de ces braves Canadiens qui courroient autrefois au combat comme à un jour de Fête. Oui, vous vous montrerez guerriers comme eux, comme eux attachés au Gouvernement sous lequel vous avez le bonheur de vivre; \& quand même il se trouveroit contre vous quelques uns de ceux qui sont descendus du même sang que vous, de ces François modernes, si prodigieusement dégénérés de leurs Peres, \& qui n'ont plus avec vous d'autre lien que celui de l'humanité; vous ne laisserez pas de les combattre \& de les vaincre, parce qu'ils seront les ennemis de votre Roi \& les vôtres.

O ! Seigneur, Dieu des batailles, vous voyez ces généreux guerriers, pleins d'une noble ardeur pour la défense de leurs familles, de leurs autels, de la Religion sainte qu'ils chérissent plus que leur propre vie; exaucez-les donc, $\hat{o}$ mon Dieu ! dans le temps de la tribulation: Exaudiat te Dominus in die tribulationis. Nos ennemis se confient présomptueusement en la force de leurs bras \& de leurs armes; mais pour nous, notre unique espérance est en votre nom: nos autem in nomine Domini Dei nostri invocabimus. Donnez donc à notre jeunesse cette valeur martiale qui méprise les dangers, à leurs Officiers cette prudence qui assure la victoire, à tous l'amour de la patrie \& de leur Roi; mais surtout cette innocence de moeurs qui attire vos bénédictions $\&$ sans laquelle tout leur courage ne feroit que les précipiter dans l'abyme. Nous vous en conjurons, Seigneur, par la victime que je vais immoler sur l'autel, \& dont le sang précieux doit être le gage de nos succès temporels. \& de la bienheureuse immortalité. Ainsi-soit-il. 